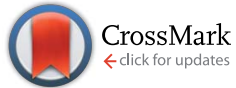

Cite this: RSC Adv., 2015, 5, 52321

DOI: $10.1039 / c 5 r a 90057 c$

www.rsc.org/advances

\title{
Correction: Electronic pH switching of DNA triplex reactions
}

\author{
Gabriel Antonio S. Minero, Patrick F. Wagler, Alaa A. Oughli and John S. McCaskill*
}

Correction for 'Electronic pH switching of DNA triplex reactions' by Gabriel Antonio S. Minero et al., RSC Adv., 2015, 5, 27313-27325.

The authors apologise for the misleading information regarding the preparation of the S-modified oligonucleotides P12 and S12, originally cited as ref. 43. The synthesis of the modified T bases for constructing oligonucleotides P12 and S12 is not discussed in ref. 43 , but it is included in a work in progress by V. Patzke et al. (unpublished, see below).

As a result, the authors wish to make the following corrections:

In the Materials and methods section, in the subsection regarding DNA sample preparation (page 27315), the last sentence is revised as: "S-modified DNA oligonucleotides P12 and S12 were chosen with the same sequences employed in ref. 40 and were synthesized by V. Patzke (Ruhr-University Bochum, Germany) ${ }^{50}$, c.f. ref. 43."

In the Results section, the first paragraph of the Triplex molecular ligation triggered electronically in microfluidic channels subsection (page 27322) should read: "The next step towards electronic DNA reaction control is to use the pH switch to control DNA-ligation on a double stranded template via triplex formation. As above, the double H-structure (Fig. 1 and 6) was chosen to perform $\mathrm{pH}$ changes for these triplex-mediated ligation experiments (Fig. 8). As a ligation chemistry, we chose the recent rapid S-S ligation scheme. ${ }^{43,50}$ Real-time monitoring of the reaction was possible using the quencher leaving group system, designed for that system, involving Alexa488 and Dabcyl (see Materials and methods). The stability of Alexa488 labeled DNA was investigated separately, and it was found that it undergoes a complete quenching transition at $\mathrm{pH} 5-4 . "$

The Acknowledgements section is amended as follows:

"The authors wish to thank Jana Bagheri-Maurer, Thomas Maeke, Tarik Abdulazim and Abhishek Sharma for their assistance with biochemical tests, microreactor fabrication, implementation and set-up. The authors also wish to thank Volker Patzke for making (prior to publication) disulphide modified oligomers available for the ligation ${ }^{50}$, and Matthias Pankau and Günter von Kiedrowski for helpful discussions and contributions to student supervision. This research has received funding from the European Union's Seventh Framework Programme for research, technological development and demonstration under grant agreements no. 222422 (ECCell) and 249032 (MATCHIT)."

The following reference is added to the Notes and references section:

50. V. Patzke and G. von Kiedrowsk, Work in preparation for publication.

The Supplementary Information of the original article has also been updated to reflect these changes.

The Royal Society of Chemistry apologises for these errors and any consequent inconvenience to authors and readers. 